\title{
Efficient Large Deformation Registration via Geodesics on a Learned Manifold of Images
}

\author{
Jihun Hamm, Christos Davatzikos, and Ragini Verma \\ Department of Radiology, University of Pennsylvania, USA \\ \{hammj, christos .davatzikos, ragini.verma\}@uphs . upenn.edu
}

\begin{abstract}
Geodesic registration methods have been used to solve the large deformation registration problems, which are hard to solve with conventional registration methods. However, analytically defined geodesics may not coincide with anatomically optimal paths of registration. In this paper we propose a novel and efficient method for large deformation registration by learning the underlying structure of the data using a manifold learning technique. In this method a large deformation between two images is decomposed into a series of small deformations along the shortest path on the graph that approximates the metric structure of data. Furthermore, the graph representation allows us to estimate the optimal group template by minimizing geodesic distances. We demonstrate the advantages of the proposed method with synthetic $2 \mathrm{D}$ images and real 3D mice brain volumes.
\end{abstract}

\section{Introduction}

The problem of registering two images is particularly challenging in the presence of large deformations between the images, as in the case of growth in pediatric studies or pathological changes. Registration between two very different anatomies is difficult mainly for two reasons. First, an objective function such as mean-squared error (MSE) or mutual information (MI) is a highly nonlinear function of the deformation variable $f$, and therefore the optimization process is often trapped in a local minimum. Second, the uncertainty about the oneto-one correspondences in the images makes the optimization problem ill-posed. Although regularization on the final deformation $f$ is commonly used to remedy the situation, the regularization alone cannot guide the variable $f$ to an optimal path of registration. Geodesic registration methods have been proposed to cope with these challenges. In the framework of [12], the optimal deformation path $f(x, t)$ is computed by solving the Euler-Lagrange equation:

$$
\frac{\partial f(x, t)}{\partial t}=v(f(x, t), t), \quad f(x, 0)=x,
$$

where $v$ is the velocity field, and $t \in[0,1]$. Among the possible solutions of the ODE, the geodesic path is defined as the 'shortest' path that connects two

\footnotetext{
^ This work was supported by NIH grants R01-MH079938 and R01-AG014971.
} 


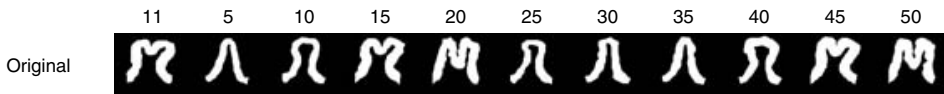

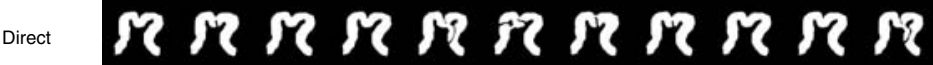

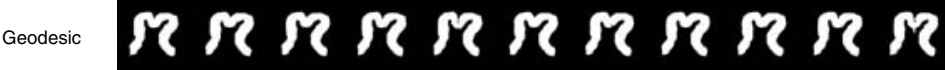

Fig. 1. Examples of synthetic images and typical registration results. The top row shows the subset of original images with the sample numbers on top. The image 11 in the first column is the template. The second and the third rows show the comparison of direct vs the proposed registration methods. Note the unnatural warping of the boundaries in the direct method, especially in image 20, 25, and 50 .

images. The length of the geodesic path is defined as a weighted sum of the intensity difference and the smoothness measure of the velocity field $v$ :

$$
g^{2}\left(I_{i}, I_{j}\right)=\int_{\Omega}\left|I_{i}(x)-I_{j}(f(x))\right|^{2} d x+w \int_{0}^{1}\|v(x, t)\|_{L}^{2} d t .
$$

When all images are in the single orbit of diffeomorphisms, the quantity $g$ provides the dataset with a true metric structure. Registration methods which are based on this numerical integration of the geodesics provide improvements in the large deformation problems [3]4. However, the increase in the computational costs can outweigh the benefits of geodesic registration methods for certain problems. More importantly, these methods calculate geodesics on the manifold of diffeomorphic transformations, most of which do not represent valid anatomies. One ideally wants to calculate geodesics on the manifold of all transformations that represent true biological variations, which unfortunately cannot be represented analytically.

We aim to achieve this goal by learning this manifold from the data. In this paper, we approximate the shortest path on the continuous space of diffeomorphisms by the shortest path on a k-nearest-neighbor $(\mathrm{kNN})$ graph that approximates the metric structure of underlying data manifold. A large deformation that maps an anatomy to another, is decomposed into a series of small deformations along the path on the graph. This means that the large deformation actually progresses through a series of small deformations between the anatomies in the data. Since only nearby images need to be registered to each other, a simple registration algorithm suffices to solve the difficult registration problem. We will call this approach a pseudo-geodesic registration method, because the registration is restricted to the paths on the assumed manifold of the data.

The advantages of the proposed registration method include the following:

1. Learning of anatomical manifolds: Our method computes the geodesics on the manifold of the actually observed anatomical variations from the data, instead of the infinite-dimensional manifold of diffeomorphisms. 
2. Efficient computation: Our method solves a large deformation problem by using simpler and faster algorithms such as the Diffeomorphic Demons algorithm [5] which we use for pairwise registrations.

3. Visualization: Our method provides a low-dimensional characterization of the data structure by flattening the learned manifold.

4. Automatic template selection: The optimal template is chosen with a criteria consistent with the pseudo-geodesics, instead of being solved iteratively as in 43 .

5. Meta-registration: Our approach is flexible to the choices of registration cost or an algorithm as its components.

The examples in Figure 1 demonstrate the accuracy of the proposed approach achievable with the same registration method that fails to find optimal solutions when used directly without the pseudo-geodesic paths.

Related Work. The pseudo-geodesic path relies on the learning methods for discovering the empirical manifold of data instead of numerical methods of solving ODEs on an analytic manifold of shapes. In our method we adopt the Isomap algorithm [6] to compute and visualize the manifold structure of data, which was originally proposed for nonlinear dimensionality reduction of high-dimensional data. Several authors have proposed the learning-based methods for template selection and visualization of the data 7,8,9. Although their goals are different from ours, these methods build the models of data by pairwise registration of multiple images similarly to our method. However, those works lack the counterpart the pseudo-geodesics which is the key to the computational efficiency of the proposed method in this paper.

The remainder of the paper is organized as follows. Section 2 describes the proposed algorithms in detail, Section 3 illustrates the application of the algorithm to $2 \mathrm{D}$ and $3 \mathrm{D}$ image databases, and Section 4 concludes the paper.

\section{Pseudo-geodesic Registration}

In this section we provide the algorithmic details of the pseudo-geodesic registration. The overall registration procedure consists of three stages which are described in the following three subsections. First, we analyze the data structure by coarse registrations between all image pairs. From this we find a kNN graph structure and a low-dimensional embedding of the data. In the second stage, we choose a template automatically from the graph structure, and identify the pseudo-geodesic paths from the template to the other samples. In the third stage, we compute the final large deformation by composing small deformations between adjacent images along the paths.

Throughout the paper, let's assume the dataset $\mathcal{I}$ consists of $n$ images $I_{1}, \ldots, I_{n}$, and each image is a nonnegative real function on a $2 \mathrm{D}$ or a $3 \mathrm{D}$ domain $\Omega$. 


\subsection{Construction of Empirical Manifolds}

In the first stage we construct the empirical manifold of data by investigating its metric structure. For this purpose we represent the data as a graph whose vertices correspond to the image samples. Below is the summary of the required steps.

1. Perform a coarse registrations between all pair of images. The minimum of the registration cost function between $I_{j}$ is $I_{i}$ after registration is considered the length $d_{i j}$ of the edge $e_{i j}$ 1]

2. Construct a connected $\mathrm{kNN}$ or $\epsilon$-NN graph based on the edge lengths.

3. Find the pseudo-geodesics (=shortest paths on the graph) between all pairs of vertices, e.g., by Dijkstra's or Floyd-Warshall algorithm. The length $g_{i j}$ of a pseudo-geodesic is the sum of its edge lengths $d_{k l}$ along the path.

4. (Optional) Visualize the Euclidean embedding of the data by solving eigenvalue problems (refer to $[6$ for details).

One can show that the shortest path length $g_{i j}$ is a valid metric by construction regardless of the properties of the constituent distance $d_{i j}$. The most timeconsuming part in practice is step 1 which requires $O\left(n^{2}\right)$ registrations between all image pairs. To reduce the overhead we perform the registration on the coarse resolution images of the original data, and also use fewer number of iterations than the final registration. This heuristic is justified by the observation that the kNN graph topology does not change much by the approximation.

\subsection{Automatic Template Selection}

An unbiased template of the given data can be defined as the geodesic mean of the data [4]3. From the graph derived in the previous section, we can choose a template from the samples that is closest to the pseudo-geodesic mean:

$$
I_{T}=\arg \min _{i} \sum_{j} g^{2}\left(I_{i}, I_{j}\right) .
$$

Since we have already computed the geodesic lengths $g_{i j}$, the template can be chosen by looking-up of the values. Two other alternatives to the mean are the center $\left(I_{T}=\arg \min _{i} \max _{j} g\left(I_{i}, I_{j}\right)\right)$ and the median $\left(I_{T}=\arg \min _{i} \sum_{j} g\left(I_{i}, I_{j}\right)\right)$ of the graph. The three templates look similar in our experiments. We choose the median as the template due to its resilience to outlier samples in the data.

\subsection{Computation of Large Deformations}

We compute the large deformation from the template $I_{T}$ to any node $I_{j}$ by a recursive composition of the small deformations from its edges along the pseudogeodesic path. Let $f_{i, j}: \Omega_{i} \rightarrow \Omega_{j}$ denote the deformation field computed from

${ }^{1}$ The cost function of a generic registration algorithm is a weighted sum of the intensity difference $\int_{\Omega}\left|I_{i}(x)-I_{j}(f(x))\right|^{2} d x$ and the smoothness measure, e.g., $\int_{\Omega} \nabla^{2} f(x) d x$. 
the registration of $I_{j}$ to $I_{i}$. Given the two fields $f_{i, j}$ and $f_{j, k}$, we can easily compute the composition field $f_{i, k}=f_{j, k} \cdot f_{i, j}: \Omega_{i} \rightarrow \Omega_{k}$ by resampling and interpolation of the two fields 2 The final deformation $\hat{f}_{T, j}$ is the refinement on the composed field $f_{T, j}$ by a few additional iterations of registration. This removes the numerical errors accumulated from the composition of deformations. Below we summarize the procedure.

1. Identify $n$ pseudo-geodesic paths from $I_{T}$ to the rest $I_{j}, \forall j \in 1, \ldots, n$.

2. Enumerate all edges $\mathcal{E}$ used in any of the shortest paths. Perform accurate registration between $\left(I_{i}, I_{j}\right), \forall e_{i j} \in \mathcal{E}$.

3. For each $j \in 1, \ldots, n$,

(a) Let $s=\left(s_{1}=T, \ldots, s_{m}=j\right)$ be the pseudo-geodesic path from $I_{T}$ to $I_{j}$.

(b) If $f_{T, j}$ is already computed then exit.

(c) Otherwise, recursively compute $f_{s_{1}, s_{m}}=f_{s_{m-1}, s_{m}} \cdot f_{s_{1}, s_{m-1}}$.

(d) Fine-tune $f_{s_{1}, s_{m}}$ by a few additional iterations of registration.

Note that we needed only coarse registration results in the previous stages, and this stage is where we actually perform accurate registrations. The step 2 may seem to be a huge computational burden at first since the number of all the edges in a graph can be a large as $n^{2}$. In fact, we only need to update the registration for $n-1$ edges, that is, no more than the number of direct registration for a conventional approach. This is due to property of the graph that the shortest paths from the template to the rest forms a spanning tree. Furthermore the registration converges faster since the two adjacent images are similar by construction.

\subsection{Discussion}

The proposed registration method is motivated by the Isomap algorithm, which proves that a true geodesic on a convex set can be approximated by a path on the kNN graph connecting the data samples. Due to the finite number of samples, the pseudo-geodesic is different from the numerically integrated geodesic in general. Moreover, the different choices of the neighborhood size $k$ in $\mathrm{kNN}$ yield different graphs and therefore different paths. However, we can show that the final large deformations are robust to the these approximations: two different pseudo-geodesic paths between the common image pair result in the same final deformation as long as each step of the paths is smooth enough to avoid any local minimum of registration.

\section{Experiments}

\subsection{Synthetic Images}

The data consist of 60 binary images of size $140 \times 140$ which simulate a patch of a cortex varying in shape and the number of folds. We use an ITK [10] version

${ }^{2}$ Two deformations should be properly composed, not simply added to each other. 

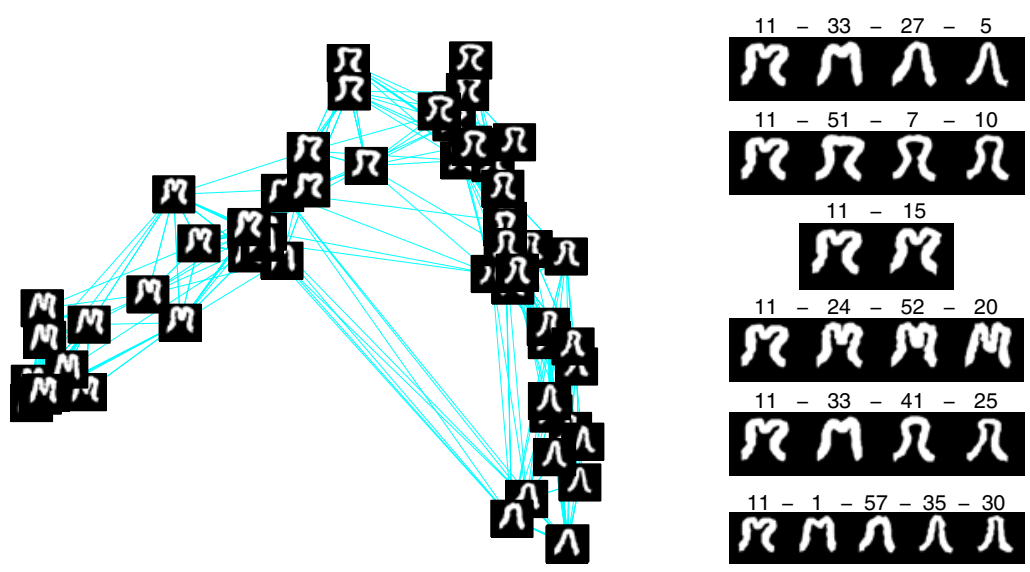

Fig. 2. Left. Two-dimensional embeddings of the manifold of synthetic shapes. The embedding reveals that there are two or three major variants (which resemble $M, \Omega, \Lambda$ ) and the rest of the images can be interpolated from those representative image. Right. Examples of the pseudo-geodesic paths from the template 11 to 5, 10, 15, 20, 25, and 30 . Note the gradual and monotonic change of shapes along each path.

Table 1. Final registration error of the synthetic images : comparison of Mean Square Error (MSE), Harmonic Energy (HE), and Maximum of Jacobian Determinant(MJD). The (avg) and $(\max )$ are the average and maximum of all images in the data.

\begin{tabular}{|l|c|c|c|c|c|c|}
\hline Method & MSE (avg) & MSE (max) & HE (avg) & HE (max) & MJD (avg) & MJD (max) \\
\hline Direct & 388 & 1136 & 0.260 & 0.583 & 4.01 & 14.6 \\
Pseudo-geodesic & 327 & 565 & 0.218 & 0.504 & 3.55 & 7.70 \\
\hline
\end{tabular}

of the Diffeomorphic Demons [5] for registration due to its efficiency. Images are registered with three levels of resolution $(10 \times 10 \times 10)$ and the smoothing kernel size of 1.5. Figure 2 shows the two-dimensional Euclidean embedding of the synthetic database and a few examples of pseudo-geodesic paths. The registration results are shown in Figure 1. For comparison we perform the direct registration of all images to the template without following the pseudo-geodesic paths. One can visually confirm the superior quality of the pseudo-geodesic registration results over the direct registration results obtained from the same algorithms and parameters. Table 1 summarizes the final registration errors. For each of the six error criteria, the pseudo-geodesic method achieves smaller error than the direct registration method does.

\subsection{Fractional Anisotropy Map of Mouse}

Next we apply our registration method to a dataset of mouse brains which is collected with the aim of creating a normative atlas of a developing mouse brain. The data consist of 69 Fractional Anisotropy volume of the brains sampled at $2,3,4,7,10,15,20,30,45$, and 80 days of age. Each volume is resized to $150 \times 150 \times 100$ 

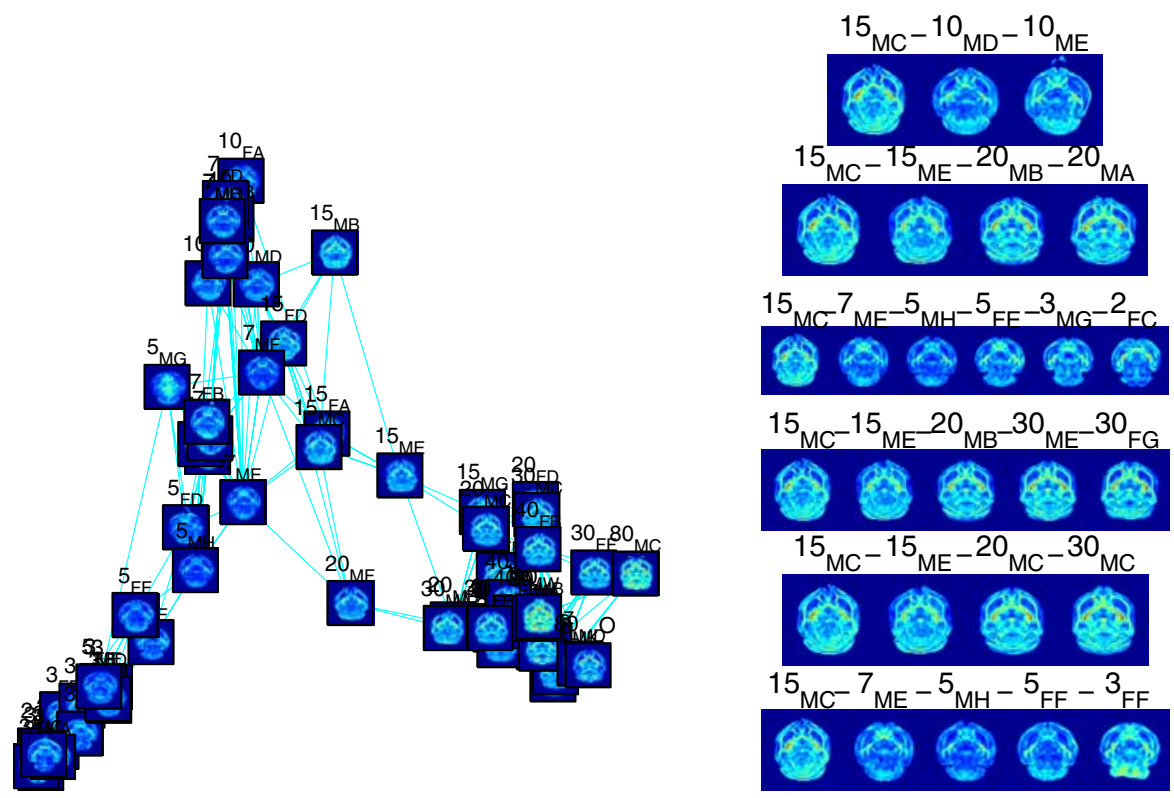

Fig. 3. Left. Two-dimensional embeddings of the manifold of mouse brains. A midaxial slice is shown for each mouse brain volume. The number on top of each image is the age and name of the sample. Right. Examples of the pseudo-geodesic paths from the template to others. Each path reflects the changes in both the shapes and the appearances of developing brains.

Table 2. Final registration error of the mouse brain images. See Table 1 for notations.

\begin{tabular}{|l|c|c|c|c|c|c|}
\hline Method & MSE (avg) & MSE (max) & HE (avg) & HE (max) & MJD (avg) & MJD (max) \\
\hline Direct & 19.33 & 40.11 & 0.0984 & 0.1927 & 9.051 & 19.92 \\
Pseudo-geodesic & 19.98 & 41.62 & 0.0800 & 0.1345 & 8.789 & 13.02 \\
\hline
\end{tabular}

and affinely aligned. We also use the Diffeomorphic Demons method for pairwise registration, with two levels of resolution $15 \times 10$ and with $\sigma=1.0$. The images in this dataset not only have a larger number of voxels than the synthetic data, but they are more challenging for registration due to their large shape and appearance variations from different ages and the degrees of maturation of tracts.

The two-dimensional embedding of the data in Figure 3 (left) provides a glimpse of its manifold structure. From the figure we can observe that the major variability of the data comes from the age. The importance of the age factor is also observed in Figure 3 (right): a path that connects two brain images of different ages passes through brains of intermediate ages in a monotonic fashion. These findings are consistent with our prior knowledge of the database. Table 2 summarizes the final registration errors. The MSE of the pseudo-geodesic method is slightly worse but comparable with the MSE of the direct method. However, 
the harmonic energy and the Jacobian determinants are significantly lower in the pseudo-geodesic method. One possible interpretation is that the pseudo-geodesic method can achieve the similar level of intensity difference as the direct method but with a much smoother deformation.

\section{Conclusion}

In this paper we propose a novel method of performing a large deformation registration. The most distinguishing feature of the method is that it computes the geodesics on the manifold of the observed anatomical variations from the data, instead of computing the geodesics by solving ODEs. Furthermore, the learned manifold provides a visualization of the data structure and allows us to choose an optimal template among the samples. We describe an meta-registration algorithm to efficiently compute large deformations, which can use a large class of registration algorithms as a component. The experiments have their caveats but show promising results. We are currently testing the algorithms on a number of other image databases. A comprehensive study will be reported in the near future.

\section{References}

1. Grenander, U., Miller, M.I.: Computational anatomy: an emerging discipline. Quart of Appl. Math., 617-694 (1998)

2. Miller, M., Trouve, A., Younes, L.: On the metrics and euler-lagrange equations of computational anatomy. Ann. Rev. of Biomed. Eng. 4, 375-405

3. Avants, B., Gee, J.C.: Geodesic estimation for large deformation anatomical shape averaging and interpolation. NeuroImage 23, S139-S150 (2004)

4. Joshi, S., Davis, B., Jomier, M., Gerig, G.: Unbiased diffeomorphic atlas construction for computational anatomy. Neuroimage (23 suppl. 1) (2004)

5. Vercauteren, T., Pennec, X., Perchant, A., Ayache, N.: Non-parametric diffeomorphic image registration with the demons algorithm. In: Ayache, N., Ourselin, S., Maeder, A. (eds.) MICCAI 2007, Part II. LNCS, vol. 4792, pp. 319-326. Springer, Heidelberg (2007)

6. Tenenbaum, J.B., de Silva, V., Langford, J.C.: A global geometric framework for nonlinear dimensionality reduction. Science 290(5500), 2319-2323 (2000)

7. Blezek, D.J., Miller, J.V.: Atlas stratification. In: Larsen, R., Nielsen, M., Sporring, J. (eds.) MICCAI 2006. LNCS, vol. 4190, pp. 712-719. Springer, Heidelberg (2006)

8. Sabuncu, M.R., Balci, S.K., Golland, P.: Discovering modes of an image population through mixture modeling. In: Metaxas, D., Axel, L., Fichtinger, G., Székely, G. (eds.) MICCAI 2008, Part II. LNCS, vol. 5242, pp. 381-389. Springer, Heidelberg (2008)

9. Rohde, G.K., Ribeiro, A.J.S., Dahl, K.N., Murphy, R.F.: Deformation-based nuclear morphometry: Capturing nuclear shape variation in hela cells. Cytometry Part A $73 \mathrm{~A}(4), 341-350$ (2008)

10. Ibanez, L., Schroeder, W., Ng, L., Cates, J.: The ITK Software Guide. Kitware, Inc. (2005), http://www.itk.org/ItkSoftwareGuide.pdf 